\title{
The Existence of Multiple Positive Solutions of a Riemann-Liouville Fractional q-Difference Equation Under Four-Point Boundary Value Condition with p-Laplacian Operator
}

\author{
Congying JIANG and Chengmin $\mathrm{HOU}^{1}$ \\ Department of Mathematics, School of Science, Yanbian University, 997 Gongyuan \\ Road, Yanji City, Jilin Province, China
}

\begin{abstract}
This paper mainly studies the existence of multiple positive solutions of a class of Riemann-Liouville fractional q-difference equations under the four-point boundary value condition with p-Laplacian operator. The existence of two positive solutions of the q-difference equation is verified by the monotonic iterative method. Finally, an example is used to prove the validity of the main results obtained.
\end{abstract}

Keywords. P-Laplacian operator, q-difference equation, multiple positive solutions, the existence.

\section{Introduction}

The calculus invented by Newton and Leibnitz is the watershed between modern mathematics and ancient mathematics. Fractional calculus is a related theory about differentiation and integration of any order. It is the extension of integer-order calculus. From q-differential calculus and quantum after the calculus was proposed by Jackson, it attracted the attention of many scholars to the q-difference equation. Quantum calculus is called infinite calculus. It replaces the classical derivative with a difference operator and can be used to calculate non-differentiable functions. In addition to the application of q-difference to orthogonal polynomials, combinatorics, hypergeometric functions and other mathematical fields, q-differences are increasingly used in natural sciences and engineering [1].

At present, fractional differential equations with p-Laplacian operators have received widespread attention due to their outstanding applications in viscoelastic mechanics, non-Newtonian mechanics, electrochemistry, fluid mechanics, and materials science. The related theoretical research on the boundary value problem of q-differential equation with p-Laplacian operator is not only the need of the development of differential equation theory, but also the need of social production and

\footnotetext{
${ }^{1}$ Corresponding Author, C M HOU, Department of Mathematics, School of Science, Yanbian University, 997 Gongyuan Road, Yanji City, Jilin Province, China; Email: cmhou@foxmail.com.
} 
life. It is hoped that its related theories can play a certain role in practical applications $[2,3]$.

In recent years, some preliminary results have been achieved:

In 2018, Bai, C. [4] studied the following problems with p-Laplacian operators:

$$
\left\{\begin{array}{c}
\left(\varphi_{p}\left(D_{0+}^{\alpha} u(t)\right)\right)^{\prime}+f(t, u(t))=0, \quad 0<t<1, \\
u(0)=D_{0+}^{\alpha} u(0)=0, \quad{ }^{C} D_{0}^{\beta} u(0)={ }^{C} D_{0}^{\beta} u(1)=0
\end{array}\right.
$$

with $0<\beta \leq 1,2<\alpha \leq 2+\beta$, and $\alpha, \beta$ are real number, $D_{0+}^{\alpha}$ and ${ }^{C} D_{0}^{\beta}$ are the standard Riemann-Liouville fractional derivative and Caputo fractional derivative of order $\alpha, \beta$ respectively, $p>1, f \in \mathrm{C}([a, b] \times R, R)$, which used to prove the existence and uniqueness of nontrivial solution with fractional boundary value using the Banach contraction mapping theorem and Guo-Krasnosel'skii fixed point theorem

In 2019, Zhou, B. [5] studied the following high-order fitting fractional boundary value problems with p-Laplacian operators:

$$
\left\{\begin{array}{c}
T_{\alpha}^{0+}\left(\varphi_{p}\left(T_{\alpha}^{0+} u(t)\right)\right)=f\left(t, u(t), T_{\alpha}^{0+} u(t)\right), \\
u^{(i)}(0)=0, \quad\left[\varphi_{p}\left(T_{\alpha}^{0+} u\right)\right]^{(i)}(0)=0, \\
{\left[T_{\beta}^{0+} u(t)\right]_{t=1}=0, \quad\left[T_{\beta}^{0+}\left(\varphi_{p}\left(T_{\alpha}^{0+} u(t)\right)\right)\right]_{t=1}=0,}
\end{array}\right.
$$

with $n-1 \leq \alpha<n, T_{\alpha}^{0+}$ is a newly defined fractional derivative called "integrated fractional derivative". Using the Guo-Krasnosel'skii fixed point theorem, sufficient conditions are established to ensure the existence of a positive solution to the above boundary value problem.

For some basic theories and applications of fractional boundary value problems with p-Laplacian operators, please refer to the literature [6-18].

In 2020, Zhou, J. et al. [19] studied the boundary value problem of fractional q-difference equations:

$$
\left\{\begin{array}{c}
D_{q}^{\alpha} u(t)+f(t, u(t), v(t))=0, \quad t \in(0,1), \\
u(0)=0, \quad D_{q} u(0)=D_{q} u(1)=0,
\end{array}\right.
$$

where $0<q<1,2<\alpha \leq 3$, the function $f(t, u, v)$ may be singular at $v=0$ and $t=0,1$ around. The iterative algorithm is used to obtain the existence and uniqueness of the positive solution of the boundary value problem.

For some basic theories and applications of boundary value problems of fractional q-difference equations, please refer to the literature [20-25].

Inspired by the above literature, we discuss the following equation:

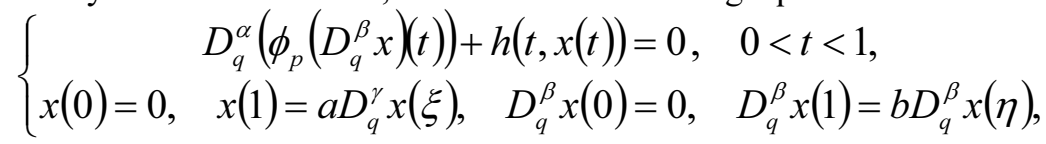


where $\quad h \in C([0,1] \times[0,+\infty),[0,+\infty)), D_{q}^{\alpha}, D_{q}^{\beta}$ and $D_{q}^{\gamma}$ stand $\quad$ for $\quad$ the

Riemann-Liouville fractional q-derivative, $\varphi_{p}$ is $\mathrm{p}$-Laplacian operator, $p>1$, $\varphi_{p}(s)=|s|^{p-1} s, \varphi_{p}^{-1}=\varphi_{q}^{*}$,

$\frac{1}{p}+\frac{1}{q^{*}}=1,1<\alpha, \beta \leq 2, \gamma=\frac{\beta-1}{2}, 0<\xi \leq \frac{1}{2}, 0<\eta<1, a, b \in[0,+\infty)$,

$a \Gamma_{q}(\beta) \xi^{\frac{\beta-1}{2}}<\Gamma_{q}\left(\frac{\beta+1}{2}\right), b^{p-1} \eta^{\alpha-1}<1$.

\section{Preliminaries}

In this section, let $q \in(0,1)$, some related definitions and lemmas are given.

Definition 2.1[26] $[m]_{q}=\frac{1-q^{m}}{1-q}, m \in R$.

Definition 2.2[26] The q-similar definition power function $(n-m)^{k}$, $k \in N_{0}:=\{0,1,2, \ldots\}$ is:

$$
(n-m)^{(0)}=1,(n-m)^{(k)}=\prod_{i=0}^{k-1}\left(n-m q^{i}\right), k \in N, m, n \in R .
$$

Generally, $\gamma \in R$,

$$
(n-m)^{(\gamma)}=n^{\gamma} \prod_{i=0}^{\infty} \frac{n-m q^{i}}{n-m q^{\gamma+i}}, n \neq 0 .
$$

Particularly, $m=0, n^{(\gamma)}=n^{\gamma},[a(n-m)]^{(\gamma)}=a^{\gamma}(n-m)^{(\gamma)}$.

Definition 2.3[26] q-gamma function is defined by

Then, $\Gamma_{q}(t+1)=[t]_{q} \Gamma_{q}(t)$.

$$
\Gamma_{q}(t)=\frac{(1-q)^{(t-1)}}{(1-q)^{t-1}}, t \in R \backslash\{0,-1,-2, \ldots\} .
$$

Definition 2.4[26] The q-derivatives for $h$ is defined by

$$
\begin{gathered}
\left(D_{q} h\right)(t)=\frac{h(t)-h(q t)}{(1-q) t}, t \neq 0 . \\
\left(D_{q} h\right)(0)=\lim _{t \rightarrow 0}\left(D_{q} h\right)(t) .
\end{gathered}
$$

Definition 2.5[26] The high order q-derivatives for $h$ is defined by

$$
\begin{gathered}
\left(D_{q}^{0} h\right)(t)=h(t), \\
\left(D_{q}^{k} h\right)(t)=D_{q}\left(D_{q}^{k-1} h\right)(t), k \in N .
\end{gathered}
$$


Definition 2.6[26] $h$ is defined on the interval $[0, b]$, q-integral for 0 to $b$ is defined by

$$
\left(I_{q} h\right)(t)=\int_{0}^{t} h(s) d_{q} s=t(1-q) \sum_{i=0}^{\infty} h\left(t q^{i}\right) q^{i}, t \in[0, b] .
$$

Lemma 2.1[27] If $a \in[0, b]$, and $h$ is defined on the interval $[0, b]$, q-integral for $a$ to $b$ is defined by

$$
\int_{a}^{b} h(s) d_{q} s=\int_{0}^{b} h(s) d_{q} s-\int_{0}^{a} h(s) d_{q} s .
$$

Lemma 2.2[27] The operator $I_{q}^{k}$ obtains

$$
\left(I_{q}^{0} h\right)(t)=h(t), \quad\left(I_{q}^{k} h\right)(t)=I_{q}\left(I_{q}^{k-1} h\right)(t), \quad k \in N .
$$

Lemma 2.3[27] $\left(D_{q} I_{q} h\right)(t)=h(t)$; if function $h$ is continue at $t=0$, $\left(I_{q} D_{q} h\right)(t)=h(t)-h(0)$.

Definition 2.7[26] Let $v \geq 0$ and $h$ be a real function defined on a certain interval $[0, T]$. The Riemann-Liouville fractional q-integral of order $v$ is defined by $\left(I_{q}^{0} h\right)(t)=h(t)$ and

$$
\left(I_{q}^{v} h\right)(t)=\frac{1}{\Gamma_{q}(v)} \int_{0}^{t}(t-q s)^{(v-1)} h(s) d_{q} s, \quad v>0, \quad t \in[0, T] .
$$

Definition 2.8[26] Let $v>0$. The Riemann-Liouville fractional q-derivative of order $v$ is defined by Riemann-Liouville $\left(D_{q}^{0} h\right)(t)=h(t)$ and

$$
\left(D_{q}^{v} h\right)(t)=\left(D_{q}^{l} I_{q}^{l-v} h\right)(t), v>0 \text {. }
$$

Where $l$ is the smallest integer greater than or equal to $v$.

Lemma 2.4[27] Let $\alpha, \beta \geq 0, f$ be a function defined on a certain interval $[0, T]$, Then the following formulas hold:

$$
\begin{aligned}
& \text { (1) }\left(I_{q}^{\beta} I_{q}^{\alpha} f\right)(t)=\left(I_{q}^{\alpha+\beta} f\right)(t), \\
& \text { (2) }\left(D_{q}^{\alpha} I_{q}^{\alpha} f\right)(t)=f(t) .
\end{aligned}
$$

Lemma 2.5[27] Let $\alpha>0, n$ is positive integer. Then the following equality hold:

$$
\left(I_{q}^{\alpha} D_{q}^{n} f\right)(t)=\left(D_{q}^{n} I_{q}^{\alpha} f\right)(t)-\sum_{i=0}^{n-1} \frac{t^{\alpha-n+i}}{\Gamma_{q}(\alpha+i-n-1)}\left(D_{q}^{i} f\right)(0) .
$$

Lemma 2.6[28] Let $v>0, \alpha \in R$, for $t \in[a, b]$, Then the following equality hold:

$$
D_{q}^{v}(t-a)^{\alpha}=\frac{\Gamma_{q}(\alpha+1)}{\Gamma_{q}(\alpha-v+1)}(t-a)^{\alpha-v} .
$$

Definition 2.9[29] Let $p>1$, the p-Laplacian operator is defined by 


$$
\varphi_{p}(x)=|x|^{p-2} x
$$

Obviously, $\varphi_{p}$ is the continuously increasing invertible operator, its inverse operator is $\varphi_{q^{*}}, q^{*}>1, \frac{1}{p}+\frac{1}{q^{*}}=1$.

Lemma 2.7 For any $y \in C[0,1]$, the problem

$$
\left\{\begin{array}{c}
D_{q}^{\alpha}\left(\phi_{p}\left(D_{q}^{\beta} x\right)(t)\right)=y(t), \quad 0<t<1, \\
x(0)=0, \quad x(1)=a D_{q}^{\gamma} x(\xi), \quad D_{q}^{\beta} x(0)=0, \quad D_{q}^{\beta} x(1)=b D_{q}^{\beta} x(\eta),
\end{array}\right.
$$

has the unique solution

$$
x(t)=\int_{0}^{1} G(t, q z) \phi_{q}\left(\int_{0}^{1} M(z, q r) y(r) d_{q} r\right) d_{q} z .
$$

With

$$
\begin{aligned}
& B_{1}=b^{p-1} \eta^{\alpha-1} \neq 1, B_{2}=a \Gamma_{q}(\beta) \xi^{\frac{\beta-1}{2}} \neq \Gamma_{q}\left(\frac{\beta+1}{2}\right), \\
& \left\{\frac{z^{\alpha-1}(1-q r)^{(\alpha-1)}-b^{p-1} z^{\alpha-1}(\eta-q r)^{(\alpha-1)}-\left(1-B_{1}\right)(z-q r)^{(\alpha-1)}}{\Gamma_{q}(\alpha)\left(1-B_{1}\right)}, \quad 0 \leq q r \leq z \leq 1, q r \leq \eta,\right. \\
& M(z, q r)= \begin{cases}\frac{z^{\alpha-1}(1-q r)^{(\alpha-1)}-\left(1-B_{1}\right)(z-q r)^{(\alpha-1)}}{\Gamma_{q}(\alpha)\left(1-B_{1}\right)}, & 0 \leq \eta \leq q r \leq z \leq 1, \\
\frac{z^{\alpha-1}(1-q r)^{(\alpha-1)}-b^{p-1} z^{\alpha-1}(\eta-q r)^{(\alpha-1)}}{\Gamma_{q}(\alpha)\left(1-B_{1}\right)}, & 0 \leq z \leq q r \leq \eta \leq 1,\end{cases} \\
& \frac{z^{\alpha-1}(1-q r)^{(\alpha-1)}}{\Gamma_{q}(\alpha)\left(1-B_{1}\right)}, \quad 0 \leq z \leq q r \leq 1, \eta \leq q r, \\
& \int \frac{\left.\Gamma_{q}\left(\frac{\beta+1}{2}\right)\right)^{\beta-1}(1-q z)(\beta-1)-\left(\Gamma_{q}\left(\frac{\beta+1}{2}\right)-B_{2}\right)(t-q z)(\beta-1)-a \Gamma_{q}(\beta) t^{\beta-1}(\xi-q z)\left(\frac{\beta-1}{2}\right)}{\Gamma_{q}(\beta)\left(\Gamma_{q}\left(\frac{\beta+1}{2}\right)-B_{2}\right)}, 0 \leq q z \leq t \leq 1, q z \leq \xi, \\
& \frac{\Gamma_{q}\left(\frac{\beta+1}{2}\right) t^{\beta-1}(1-q z)(\beta-1)-\left(\Gamma_{q}\left(\frac{\beta+1}{2}\right)-B_{2}\right)(t-q z)(\beta-1)}{\Gamma_{q}(\beta)\left(\Gamma_{q}\left(\frac{\beta+1}{2}\right)-B_{2}\right)}, 0<\xi \leq q z \leq t \leq 1, \\
& \frac{\Gamma_{q}\left(\frac{\beta+1}{2}\right) t^{\beta-1}(1-q z)(\beta-1)-a \Gamma_{q}(\beta) t^{\beta-1}(\xi-q z)}{\Gamma_{q}(\beta)\left(\Gamma_{q}\left(\frac{\beta+1}{2}\right)-B_{2}\right)}, 0 \leq t \leq q z \leq \xi<1, \\
& \frac{\Gamma_{q}\left(\frac{\beta+1}{2}\right) t^{\beta-1}(1-q z)(\beta-1)}{\Gamma_{q}(\beta)\left(\Gamma_{q}\left(\frac{\beta+1}{2}\right)-B_{2}\right)}, 0 \leq t \leq q z \leq 1, \xi \leq q z .
\end{aligned}
$$

Proof By Lemma 2.5, one has

$$
\begin{gathered}
I_{q}^{\alpha} D_{q}^{\alpha}\left(\phi_{p}\left(D_{q}^{\beta} x(t)\right)\right)=\phi_{p}\left(D_{q}^{\beta} x(t)\right)-A_{1} t^{\alpha-1}-A_{2} t^{\alpha-2} \\
=I_{q}^{\alpha} y(t) \\
=\frac{1}{\Gamma_{q}(\alpha)} \int_{0}^{1}(t-q r)^{(\alpha-1)} y(r) d_{q} r
\end{gathered}
$$

where $A_{1}, A_{2} \in R$. Combining (2) with $D_{q}^{\beta} x(0)=0$, we have $A_{2}=0$. Then 


$$
\phi_{p}\left(D_{q}^{\beta} x(t)\right)=\frac{1}{\Gamma_{q}(\alpha)} \int_{0}^{t}(t-q r)^{(\alpha-1)} y(r) d_{q} r+A_{1} t^{\alpha-1}
$$

Hence

$$
\begin{gathered}
\phi_{p}\left(D_{q}^{\beta} x(1)\right)=\frac{1}{\Gamma_{q}(\alpha)} \int_{0}^{1}(1-q r)^{(\alpha-1)} y(r) d_{q} r+A_{1}, \\
\phi_{p}\left(D_{q}^{\beta} x(\eta)\right)=\frac{1}{\Gamma_{q}(\alpha)} \int_{0}^{\eta}(\eta-q r)^{(\alpha-1)} y(r) d_{q} r+A_{1} \eta^{\alpha-1},
\end{gathered}
$$

combining with $D_{q}^{\beta} x(1)=b D_{q}^{\beta} x(\eta)$, one has

$$
\begin{gathered}
A_{1}=-\int_{0}^{1} \frac{(1-q r)^{(\alpha-1)}}{\Gamma_{q}(\alpha)\left(1-b^{p-1} \eta^{\alpha-1}\right)^{y}} y(r) d r+\int_{0}^{\eta} \frac{b^{p-1}(\eta-q r)^{(\alpha-1)}}{\Gamma_{q}(\alpha)\left(1-b^{p-1} \eta^{\alpha-1}\right)} y(r) d r, \\
\phi\left(D_{q}^{\beta} x(t)\right)=\frac{1}{\Gamma_{q}(\alpha)} \int_{0}^{t}(t-q r)^{(\alpha-1)} y(r) d_{q} r-\int_{0}^{1} \frac{t^{\alpha-1}(1-q r)^{(\alpha-1)}}{\Gamma_{q}(\alpha)\left(1-b^{p-1} \eta^{\alpha-1}\right)} y(r) d r \\
+\int_{0}^{\eta} \frac{b^{p-1} t^{\alpha-1}(\eta-q r)^{(\alpha-1)}}{\Gamma_{q}(\alpha)\left(1-b^{p-1} \eta^{\alpha-1}\right)} y(r) d r \\
=-\int_{0}^{1} M(t, q r) y(r) d_{q} r .
\end{gathered}
$$

So

$$
D_{q}^{\beta} x(t)=-\phi_{q^{*}}\left(\int_{0}^{1} M(t, q r) y(r) d_{q} r\right) .
$$

Applying Lemma 2.5 to (5), we have

$$
\begin{aligned}
I_{q}^{\beta} D_{q}^{\beta} x(t) & =x(t)-C_{1} t^{\beta-1}-C_{2} t^{\beta-2} \\
\quad= & -I_{q}^{\beta} \phi_{q^{*}}\left(\int_{0}^{1} M(z, q r) y(r) d_{q} r\right),
\end{aligned}
$$

where $C_{1}, C_{2} \in R$. Since $x(0)=0$, we have $C_{2}=0$. Therefore,

$$
x(t)=-I_{q}^{\beta} \phi_{q^{*}}\left(\int_{0}^{1} M(z, q r) y(r) d_{q} r\right)+C_{1} t^{\beta-1} .
$$

Applying $D_{q}^{\gamma}$ to both sides of (6), and by Lemma 2.6, we have

$$
\begin{gathered}
D_{q}^{\gamma} x(t)=-D_{q}^{\gamma} I_{q}^{\beta} \phi_{q^{*}}\left(\int_{0}^{1} M(z, q r) y(r) d_{q} r\right)+C_{1} D_{q}^{\gamma} t^{\beta-1} \\
=-\int_{0}^{t} \frac{(t-q z)\left(\frac{\beta+1}{2}\right)}{\Gamma_{q}\left(\frac{\beta+1}{2}\right)} \phi_{q^{*}}\left(\int_{0}^{1} M(z, q r) y(r) d_{q} r\right) d_{q} z+C_{1} \frac{\Gamma_{q}(\beta)}{\Gamma_{q}(\beta-\gamma)} t^{\beta-\gamma-1} .
\end{gathered}
$$

So

$$
\begin{gathered}
x(1)=-\int_{0}^{1} \frac{(1-q z)^{(\beta-1)}}{\Gamma_{q}(\beta)} \phi_{q^{*}}\left(\int_{0}^{1} M(z, q r) y(r) d_{q} r\right) d_{q} z+C_{1}, \\
D_{q}^{\gamma} x(\xi)=-\int_{0}^{\xi} \frac{(\xi-q z)^{\left(\frac{\beta+1}{2}\right)}}{\Gamma_{q}\left(\frac{\beta+1}{2}\right)} \phi_{q^{*}}\left(\int_{0}^{1} M(z, q r) y(r) d_{q} r\right) d_{q} z+C_{1} \frac{\Gamma_{q}(\beta)}{\Gamma_{q}(\beta-\gamma)} \xi^{\beta-\gamma-1},
\end{gathered}
$$


combining with $x(1)=a D_{q}^{\gamma} x(\xi)$, we have

$$
\begin{aligned}
C_{1}= & \frac{\Gamma_{q}\left(\frac{\beta+1}{2}\right)}{\Gamma_{q}\left(\frac{\beta+1}{2}\right)-B_{2}}\left\{\int_{0}^{1} \frac{(1-q z)^{(\beta-1)}}{\Gamma_{q}(\beta)} \phi_{q^{*}}\left(\int_{0}^{1} M(z, q r) y(r) d_{q} r\right) d_{q} z\right. \\
& \left.-a \int_{0}^{\xi} \frac{(\xi-q z)\left(\frac{\beta-1}{2}\right)}{\Gamma_{q}\left(\frac{\beta+1}{2}\right)} \phi_{q^{*}}\left(\int_{0}^{1} M(z, q r) y(r) d_{q} r\right) d_{q} z\right\} .
\end{aligned}
$$

Thus, we obtain the unique solution of problem (2):

$$
\begin{gathered}
x(t)=-\int_{0}^{t} \frac{(t-q z)^{(\beta-1)}}{\Gamma_{q}(\beta)} \phi_{q^{*}}\left(\int_{0}^{1} M(z, q r) y(r) d_{q} r\right) d_{q} z \\
+\frac{t^{\beta-1} \Gamma_{q}\left(\frac{\beta+1}{2}\right)}{\Gamma_{q}\left(\frac{\beta+1}{2}\right)-B_{2}}\left\{\int_{0}^{1} \frac{(1-q z)^{(\beta-1)}}{\Gamma_{q}(\beta)} \phi_{q^{*}}\left(\int_{0}^{1} M(z, q r) y(r) d_{q} r\right) d_{q^{\prime}} z\right. \\
\left.-a \int_{0}^{\xi} \frac{(\xi-q z)^{\left(\frac{\beta-1}{2}\right)}}{\Gamma_{q}\left(\frac{\beta+1}{2}\right)} \phi_{q^{*}}\left(\int_{0}^{1} M(z, q r) y(r) d_{q} r\right) d_{q} z\right\}
\end{gathered}
$$

The proof of Lemma 2.7 is complete.

Lemma 2.8 Let $G$ and $M$ be defined by (3) and (4), respectively. If $a \Gamma_{q}(\beta) \xi^{\frac{\beta-1}{2}}<\Gamma_{q}\left(\frac{\beta+1}{2}\right)$ and $b^{p-1} \eta^{\alpha-1}<1$, then:

(a) $G, M \in C([0,1] \times[0,1])$;

(b) $G(t, q z)>0, M(t, q z)>0$ for all $t, z \in(0,1)$;

(c) there exist two positive functions $\mu, v \in C((0,1),(0,+\infty))$, so that for all $z \in(0,1)$, one has

$$
\mu(q z) \geq \max _{0 \leq t \leq 1} G(t, q z), \quad v(q z) \geq \max _{0 \leq t \leq 1} M(t, q z) .
$$

Proof $(a)$ Obviously, $G(t, q z)$ and $M(t, q z)$ is a continuous function of above. $[0,1] \times[0,1]$.

(b) To prove that $G(t, q z)>0$ for all $t, z \in(0,1)$, put

$$
g_{1}(t, q z)=\frac{t^{\beta-1}(1-q z)^{(\beta-1)}-(t-q z)^{(\beta-1)}}{\Gamma_{q}(\beta)}
$$




$$
\begin{gathered}
=\frac{t^{\beta-1}(1-q z)^{(\beta-1)}-t^{\beta-1}\left(1-\frac{q z}{t}\right)^{(\beta-1)}}{\Gamma_{q}(\beta)} \\
>0, \\
g_{2}(t, q z)=\frac{t^{\beta-1}(1-q z)^{(\beta-1)}}{\Gamma_{q}(\beta)} \\
>0 .
\end{gathered}
$$

$\forall \xi \in\left(0, \frac{1}{2}\right], q z \in[0, \xi]$, we have

$$
\begin{aligned}
g^{*}(\xi, q z) & =\xi^{\frac{\beta-1}{2}}(1-q z)^{(\beta-1)}-(\xi-q z)\left(\frac{\beta-1}{2}\right) \\
& =\xi^{\frac{\beta-1}{2}}(1-q z)^{(\beta-1)}-\xi^{\frac{\beta-1}{2}}\left(1-\frac{q z}{\xi}\right)^{\left(\frac{\beta-1}{2}\right)} \\
& \geq \xi^{\frac{\beta-1}{2}}(1-q z)^{\left(\frac{\beta-1}{2}\right)}-\xi^{\frac{\beta-1}{2}}\left(1-\frac{q z}{\xi}\right)^{\left(\frac{\beta-1}{2}\right)} \\
& \geq 0 .
\end{aligned}
$$

the case $0 \leq q z \leq t \leq 1, z \leq \xi$, then

$$
\begin{aligned}
G(t, q z) & =\frac{\Gamma_{q}\left(\frac{\beta+1}{2}\right) t^{\beta-1}(1-q z)^{(\beta-1)}-\left(\Gamma_{q}\left(\frac{\beta+1}{2}\right)-B_{2}\right)(t-q z)^{(\beta-1)}-a \Gamma_{q}(\beta) t^{\beta-1}(\xi-q z)\left(\frac{\beta-1}{2}\right)}{\Gamma_{q}(\beta)\left(\Gamma_{q}\left(\frac{\beta+1}{2}\right)-B_{2}\right)} \\
& =\frac{t^{\beta-1}(1-q z)^{(\beta-1)}-(t-q z)^{(\beta-1)}}{\Gamma_{q}(\beta)}+\frac{B_{2} t^{\beta-1}(1-q z)^{(\beta-1)}-a \Gamma_{q}(\beta) t^{\beta-1}(\xi-q z)\left(\frac{\beta-1}{2}\right)}{\Gamma_{q}(\beta)\left(\Gamma_{q}\left(\frac{\beta+1}{2}\right)-B_{2}\right)} \\
& =g_{1}(t, q z)+\frac{a t^{\beta-1}}{\left(\Gamma_{q}\left(\frac{\beta+1}{2}\right)-B_{2}\right)} g^{*}(\xi, q z) \\
& >0 .
\end{aligned}
$$

The remaining three cases $0<\xi \leq z \leq t \leq 1 \quad$ or $0 \leq t \leq z \leq \xi<1 \quad$ or $0 \leq t \leq z \leq 1, \xi \leq z$ can be handled in a similar way, so that we omit the obvious modification. Thus, $G(t, q z)>0$ for all $t, z \in(0,1)$. Similarly, to prove that $M(t, q z)>0$, for all $t, z \in(0,1)$.Put

$$
\begin{aligned}
m_{1}(t, q z) & =\frac{t^{\alpha-1}(1-q z)^{(\alpha-1)}-(t-q z)^{(\alpha-1)}}{\Gamma_{q}(\alpha)} \\
= & \frac{t^{\alpha-1}(1-q z)^{(\alpha-1)}-t^{\alpha-1}\left(1-\frac{q z}{t}\right)^{(\alpha-1)}}{\Gamma_{q}(\alpha)}
\end{aligned}
$$




$$
\begin{aligned}
>0 . & \\
m_{2}(t, q z) & =\frac{t^{\alpha-1}(1-q z)^{(\alpha-1)}}{\Gamma_{q}(\alpha)} \\
& >0 .
\end{aligned}
$$

The case $0 \leq q z \leq t \leq 1, q z \leq \eta$, then

$$
\begin{gathered}
M(t, q z)=\frac{t^{\alpha-1}(1-q z)^{(\alpha-1)}-b^{p-1} t^{\alpha-1}(\eta-q z)^{(\alpha-1)}-\left(1-B_{1}\right)(t-q z)^{(\alpha-1)}}{\Gamma_{q}(\alpha)\left(1-B_{1}\right)} \\
=\frac{t^{\alpha-1}(1-q z)^{(\alpha-1)}-(t-q z)^{(\alpha-1)}}{\Gamma_{q}(\alpha)}+\frac{b^{p-1} t^{\alpha-1}\left[\eta^{\alpha-1}(1-q z)^{(\alpha-1)}-(\eta-q z)^{(\alpha-1)}\right]}{\Gamma_{q}(\alpha)\left(1-B_{1}\right)} \\
=m_{1}(t, q z)+\frac{b^{p-1} t^{\alpha-1}}{1-B_{1}} m_{1}(\eta, q z) \\
>0 .
\end{gathered}
$$

One can apply a similar argument in order to treat the remaining three cases $0 \leq \eta \leq q z \leq t \leq 1$ or $0 \leq t \leq q z \leq \eta \leq 1$ or $0 \leq t \leq q z \leq 1, \eta \leq q z$. Thus, that $M(t, q z)>0$, for all $t, z \in(0,1)$.

(c) For a fixed $q z$, the functions given by (7) and (8), respectively, are increasing in $t$ for $t \leq q z$ and decreasing in $t$ for $t \geq q z$. Therefore,

$$
\begin{aligned}
& \max _{0 \leq t \leq 1}\left\{g_{1}(t, q z), g_{2}(t, q z)\right\}=g_{1}(q z, q z)=\frac{z^{\beta-1}(1-q z)^{(\beta-1)}}{\Gamma_{q}(\beta)}, \quad q z \in(0,1) ; \\
& \max _{0 \leq t \leq 1}\left\{m_{1}(t, q z), m_{2}(t, q z)\right\}=m_{1}(q z, q z)=\frac{z^{\beta-1}(1-q z)^{(\alpha-1)}}{\Gamma_{q}(\alpha)}, \quad q z \in(0,1) .
\end{aligned}
$$

Put

$$
\begin{gathered}
\mu(q z)=g_{1}(q z, q z)+\frac{B_{2}(1-q z)^{(\beta-1)}}{\Gamma_{q}(\beta)\left(\Gamma_{q}\left(\frac{\beta+1}{2}\right)-B_{2}\right)}, \quad q z \in(0,1)^{\prime} \\
v(q z)=m_{1}(q z, q z)+\frac{B_{2}(1-q z)^{(\alpha-1)}}{\Gamma_{q}(\alpha)\left(\Gamma_{q}\left(\frac{\alpha+1}{2}\right)-B_{2}\right)}, \quad q z \in(0,1)^{\circ}
\end{gathered}
$$

It is certain that $\mu, v \in C((0,1),(0,+\infty))$.

Consider the four cases.

If $0 \leq q z \leq t \leq 1, z \leq \xi$, then

$$
\max _{0 \leq t \leq 1} G(t, q z)=\max _{0 \leq t \leq 1}\left(g_{1}(t, q z)+\frac{B_{2} t^{\beta-1}(1-q z)^{(\beta-1)}-a \Gamma_{q}(\beta) t^{\beta-1}(\xi-q z)\left(\frac{\beta-1}{2}\right)}{\Gamma_{q}(\beta)\left(\Gamma_{q}\left(\frac{\beta+1}{2}\right)-B_{2}\right)}\right)
$$




$$
\begin{aligned}
\leq g_{1}(q z, q z)+ & \frac{B_{2}(1-q z)^{(\beta-1)}}{\Gamma_{q}(\beta)\left(\Gamma_{q}\left(\frac{\beta+1}{2}\right)-B_{2}\right)} \\
& =\mu(q z) .
\end{aligned}
$$

If $0<\xi \leq z \leq t \leq 1$, then

$$
\begin{gathered}
\max _{0 \leq t \leq 1} G(t, q z)=\max _{0 \leq t \leq 1} \frac{\Gamma_{q}\left(\frac{\beta+1}{2}\right) t^{\beta-1}(1-q z)^{(\beta-1)}-\left(\Gamma_{q}\left(\frac{\beta+1}{2}\right)-B_{2}\right)(t-q z)^{(\beta-1)}}{\Gamma_{q}(\beta)\left(\Gamma_{q}\left(\frac{\beta+1}{2}\right)-B_{2}\right)} \\
=\max _{0 \leq t \leq 1}\left(\begin{array}{c}
\left.\frac{t^{\beta-1}(1-q z)^{(\beta-1)}}{\Gamma_{q}(\beta)}+\frac{B_{2} t^{\beta-1}(1-q z)^{(\beta-1)}}{\Gamma_{q}\left(\frac{\beta+1}{2}\right)-B_{2}}-\frac{(t-q z)^{(\beta-1)}}{\Gamma_{q}(\beta)}\right) \\
=g_{1}(q z, q z)+
\end{array}\right) \\
=\frac{\Gamma_{q}(\beta)\left(\Gamma_{q}\left(\frac{\beta+1}{2}\right)-B_{2}\right)}{\mu(q z) .}
\end{gathered}
$$

If $0 \leq t \leq z \leq \xi<1$, then

$$
\begin{aligned}
& \max _{0 \leq t \leq 1} G(t, q z)=\max _{0 \leq t \leq 1} \frac{\Gamma_{q}\left(\frac{\beta+1}{2}\right) t^{\beta-1}(1-q z)^{(\beta-1)}-a \Gamma_{q}(\beta) t^{\beta-1}(\xi-q z)\left(\frac{\beta-1}{2}\right)}{\Gamma_{q}(\beta)\left(\Gamma_{q}\left(\frac{\beta+1}{2}\right)-B_{2}\right)} \\
& =\max _{0 \leq t \leq 1}\left(\frac{t^{\beta-1}(1-q z)^{(\beta-1)}}{\Gamma_{q}(\beta)}+\frac{B_{2} t^{\beta-1}(1-q z)^{(\beta-1)}-a \Gamma_{q}(\beta) t^{\beta-1}(\xi-q z)\left(\frac{\beta-1}{2}\right)}{\Gamma_{q}(\beta)\left(\Gamma_{q}\left(\frac{\beta+1}{2}\right)-B_{2}\right)}\right) \\
& =g_{1}(q z, q z)+\frac{B_{2}(1-q z)^{(\beta-1)}}{\Gamma_{q}(\beta)\left(\Gamma_{q}\left(\frac{\beta+1}{2}\right)-B_{2}\right)} \\
& =\mu(q z) \text {. }
\end{aligned}
$$

If $0 \leq t \leq z \leq 1, \xi \leq z$, then

$$
\max _{0 \leq t \leq 1} G(t, q z)=\max _{0 \leq t \leq 1} \frac{\Gamma_{q}\left(\frac{\beta+1}{2}\right) t^{\beta-1}(1-q z)^{(\beta-1)}}{\Gamma_{q}(\beta)\left(\Gamma_{q}\left(\frac{\beta+1}{2}\right)-B_{2}\right)}
$$




$$
\begin{gathered}
=\max _{0 \leq t \leq 1}\left(\frac{t^{\beta-1}(1-q z)^{(\beta-1)}}{\Gamma_{q}(\beta)}+\frac{B_{2} t^{\beta-1}(1-q z)^{(\beta-1)}}{\Gamma_{q}(\beta) \Gamma_{q}\left(\frac{\beta+1}{2}\right)-B_{2}}\right) \\
=g_{1}(q z, q z)+\frac{B_{2}(1-q z)^{(\beta-1)}}{\Gamma_{q}(\beta)\left(\Gamma_{q}\left(\frac{\beta+1}{2}\right)-B_{2}\right)} \\
=\mu(q z) .
\end{gathered}
$$

Thus,

$$
\max _{0 \leq t \leq 1} G(t, q z) \leq \mu(q z), \quad q z \in(0,1) .
$$

Similarly, consider the four cases of the function $v$.

If $0 \leq q z \leq t \leq 1, q z \leq \eta$, then

$$
\begin{gathered}
\max _{0 \leq t \leq 1} M(t, q z)=\max _{0 \leq t \leq 1}\left(m_{1}(t, q z)+\frac{b^{p-1} t^{\alpha-1}\left[\eta^{\alpha-1}(1-q z)^{(\alpha-1)}-(\eta-q z)^{(\alpha-1)}\right]}{\Gamma_{q}(\alpha)\left(1-B_{1}\right)}\right) \\
\leq m_{1}(q z, q z)+\frac{B_{2}(1-q z)^{(\alpha-1)}}{\Gamma_{q}(\alpha)\left(1-B_{1}\right)} \\
=v(q z) .
\end{gathered}
$$

If $0 \leq \eta \leq q z \leq t \leq 1$, then

$$
\begin{gathered}
\max _{0 \leq t \leq 1} M(t, q z)=\max _{0 \leq t \leq 1}\left(\frac{t^{\alpha-1}(1-q z)^{(\alpha-1)}-\left(1-B_{1}\right)(t-q z)^{(\alpha-1)}}{\Gamma_{q}(\alpha)\left(1-B_{1}\right)}\right) \\
\leq \max _{0 \leq t \leq 1}\left(m_{1}(t, q z)+\frac{B_{1} t^{\alpha-1}(1-q z)^{(\alpha-1)}}{\Gamma_{q}(\alpha)\left(1-B_{1}\right)}\right) \\
\leq m_{1}(q z, q z)+\frac{B_{2}(1-q z)^{(\alpha-1)}}{\Gamma_{q}(\alpha)\left(1-B_{1}\right)} \\
=v(q z) .
\end{gathered}
$$

If $0 \leq t \leq q z \leq \eta \leq 1$, then

$$
\begin{gathered}
\max _{0 \leq t \leq 1} M(t, q z)=\max _{0 \leq t \leq 1}\left(\frac{t^{\alpha-1}(1-q z)^{(\alpha-1)}-b^{p-1} t^{\alpha-1}(\eta-q z)^{(\alpha-1)}}{\Gamma_{q}(\alpha)\left(1-B_{1}\right)}\right) \\
=\max _{0 \leq t \leq 1}\left(\frac{t^{\alpha-1}(1-q z)^{(\alpha-1)}}{\Gamma_{q}(\alpha)}+\frac{b^{p-1} t^{\alpha-1}\left[\eta^{\alpha-1}(1-q z)^{(\alpha-1)}-(\eta-q z)^{(\alpha-1)}\right]}{\Gamma_{q}(\alpha)\left(1-B_{1}\right)}\right) \\
\leq m_{1}(q z, q z)+\frac{B_{2}(1-q z)^{(\alpha-1)}}{\Gamma_{q}(\alpha)\left(1-B_{1}\right)} \\
=v(q z) .
\end{gathered}
$$

If $0 \leq t \leq q z \leq 1, \eta \leq q z$, then 


$$
\begin{gathered}
\max _{0 \leq t \leq 1} M(t, q z)=\max _{0 \leq t \leq 1} \frac{t^{\alpha-1}(1-q z)^{(\alpha-1)}}{\Gamma_{q}(\alpha)\left(1-B_{1}\right)} \\
=\max _{0 \leq t \leq 1}\left(\frac{t^{\alpha-1}(1-q z)^{(\alpha-1)}}{\Gamma_{q}(\alpha)}+\frac{B_{1} t^{\alpha-1}(1-q z)^{(\alpha-1)}}{\Gamma_{q}(\alpha)\left(1-B_{1}\right)}\right) \\
\leq m_{1}(q z, q z)+\frac{B_{2}(1-q z)^{(\alpha-1)}}{\Gamma_{q}(\alpha)\left(1-B_{1}\right)} \\
=v(q z) .
\end{gathered}
$$

Thus,

$$
\max _{0 \leq t \leq 1} M(t, q z) \leq v(q z), \quad q z \in(0,1) .
$$

The proof is complete.

Lemma 2.9 Let $E=C[0,1]$ be a continuous function space equipped with standard sup-norm $\|x\|=\max _{0 \leq t \leq 1}|x(t)|$ and denote by $P=\{x \in E \mid x(t) \geq 0,0 \leq t \leq 1\}$ the corresponding cone. Let $T: P \rightarrow E$ be given by

$$
T x(t)=\int_{0}^{1} G(t, q z) \phi_{q^{*}}\left(\int_{0}^{1} M(z, q r) h(r, x(r)) d_{q} r\right) d_{q} z,
$$

where $h \in C([0,1] \times[0,+\infty),[0,+\infty))$ and $G(t, q z)$ and $M(z, q r)$ are defined by (3) and (4), respectively. Then $T$ takes $P$ into itself, and as such is completely continuous.

Proof Since $G, M$ and $h$ are nonnegative and continuous, one has $T(P) \subset P$ and $T$ is continuous. In order to prove the complete continuity of $T$, it is necessary to use a standard argument based on the Arzela-Ascoli theorem and the Lebesgue rule of convergence theorem.

\section{Main result}

We can now formulate our main results. To this end, denote

$$
J^{-1}=\int_{0}^{1} \mu(q z) \phi_{q^{*}}\left(\int_{0}^{1} v(q z) d_{q} r\right) d_{q} z
$$

where $\mu, v$ defined in Lemma 2.8 .

Theorem 3.1 Let $h \in C([0,1] \times[0,+\infty),[0,+\infty))$, suppose there is a positive number $k$ satisfying the following:

$\left(S_{1}\right)$ if $0 \leq t \leq 1,0 \leq s_{1} \leq s_{2} \leq k$, establishment $h\left(t, s_{1}\right) \leq h\left(t, s_{2}\right)$;

$\left(S_{2}\right) \max _{0 \leq t \leq 1} h(t, k) \leq \phi_{p}(k J)$;

$\left(S_{3}\right) 0 \leq t \leq 1$ for all $h(t, 0)=0$. 
Then the problem (1) has two positive solutions $x^{*}$ and $y^{*}$ makes

(i) $0<\left\|x^{*}\right\| \leq k, \lim _{n \rightarrow \infty} T^{n} x_{0}=x^{*}$, where $x_{0}(t)=k$ for $0 \leq t \leq 1$;

(ii) $0<\left\|y^{*}\right\| \leq k, \lim _{n \rightarrow \infty} T^{n} y_{0}=y^{*}$, where $y_{0}(t)=0$ for $0 \leq t \leq 1$.

Proof Let $\Omega=\{x \in P\|x\| \leq k\}$. Suppose, $x \in \Omega$. Obviously, $0 \leq x(t) \leq\|x\| \leq k$.

From the assumptions $\left(S_{1}\right)$ and $\left(S_{2}\right)$ :

$$
0 \leq h(t, x(t)) \leq h(t, k) \leq \max _{0 \leq t \leq 1} h(t, k) \leq \phi_{p}(k J) .
$$

We claim that $T(\Omega) \subseteq \Omega$. Actually, for $\forall x \in \Omega$, we have $T x \in P$, and by Lemma 2.8, we know

$$
\begin{aligned}
\|T x\|=\max _{0 \leq t \leq 1}\left|\int_{0}^{1} G(t, q z) \phi_{q^{*}}\left(\int_{0}^{1} M(s, q r) h(r, x(r)) d_{q} r\right) d_{q} z\right| \\
\leq \int_{0}^{1} \mu(q z) \phi_{q^{*}}\left(\int_{0}^{1} v(q r) \phi_{p}(k J) d_{q} r\right) d_{q} z \\
\quad=k J \int_{0}^{1} \mu(q z) \phi_{q^{*}}\left(\int_{0}^{1} v(q r) d_{q} r\right) d_{q} z \\
=k .
\end{aligned}
$$

Thus, $T x \in \Omega$. The next proofs the existence of $x^{*}$. Take the function $x_{0} \equiv k$ on $0 \leq t \leq 1$, then $\left\|x_{0}\right\|=k$ and $x_{1}(t)=T x_{0}(t)$ with $x_{0} \in \Omega$.Define

$$
x_{n+1}=T x_{n}=T^{n+1} x_{0}, n=0,1,2, \ldots
$$

Then, for all $n=0,1,2, \ldots$,one has $x_{n} \in \Omega$.

From the assumptions $\left(S_{2}\right)$ and Lemma 2.8, we can get that for $\forall t \in[0,1]$ :

$$
\begin{aligned}
x_{1}(t)=T x_{0}(t)= & \int_{0}^{1} G(t, q z) \phi_{q^{*}}\left(\int_{0}^{1} M(z, q r) h\left(r, x_{0}(r)\right) d_{q} r\right) d_{q} z \\
& \leq \int_{0}^{1} \mu(q z) \phi_{q^{*}}\left(\int_{0}^{1} v(q r) \phi_{p}(k J) d_{q} r\right) d_{q} z \\
& \leq k J \int_{0}^{1} \mu(q z) \phi_{q^{*}}\left(\int_{0}^{1} v(q r) d_{q} r\right) d_{q} z \\
& =k=x_{0}(t) .
\end{aligned}
$$

Hence,

$$
x_{2}(t)=T x_{1}(t) \leq T x_{0}(t)=x_{1}(t), 0 \leq t \leq 1
$$

By mathematical induction, we know

$$
x_{n+1}(t) \leq x_{n}(t), 0 \leq t \leq 1, n=0,1,2, \ldots
$$


From Lemma 2.9, we know that the operator is a completely continuous operator, so $\overline{T(\Omega)}$ is a compact set.

Therefore, $\left\{x_{n}\right\}_{n=1}^{\infty}$ exists the sub-sequence converges $\left\{x_{n_{i}}\right\}_{i=0}^{\infty}$ to $x^{*} \in \Omega$. Because $\left\{x_{n_{i}}\right\}_{i=0}^{\infty}$ is monotonic, and $x_{n} \rightarrow x^{*}$, again from the continuity of the operator $T$, it can be known that $T x_{n}=x_{n+1} \rightarrow x^{*}$, that is $T x^{*}=x^{*}$.

It can be proved $T y^{*}=y^{*}$ by the same method. Take the function $y_{0} \equiv 0$, identically on $0 \leq t \leq 1$, Clearly,then $\left\|y_{0}\right\|=0$, and $y_{0} \in \Omega$.Also, $y_{1}(t)=T y_{0}(t)$.Define

$$
y_{n+1}=T y_{n}=T^{n+1} y_{0}, n=0,1,2, \ldots
$$

Then, for all $n=0,1,2, \ldots$, one has $y_{n} \in \Omega$. By the same computation as above,

From the assumptions $\left(S_{2}\right)$ and Lemma 2.8, we can see that for lemma 2.8, for $\forall t \in[0,1]$,

$$
\begin{aligned}
y_{1}(t)=T y_{0}(t)= & \int_{0}^{1} G(t, q z) \phi_{q^{*}}\left(\int_{0}^{1} M(z, q r) h\left(r, y_{0}(r)\right) d_{q} r\right) d_{q} z \\
& =\int_{0}^{1} G(t, q z) \phi_{q^{*}}\left(\int_{0}^{1} M(z, q r) h(r, 0) d_{q} r\right) d_{q} z \\
& =0=y_{0}(t) .
\end{aligned}
$$

Hence,

$$
y_{2}(t)=T y_{1}(t) \leq T y_{0}(t)=y_{1}(t), 0 \leq t \leq 1
$$

By mathematical induction, there is

$$
y_{n+1}(t) \leq y_{n}(t), 0 \leq t \leq 1, n=0,1,2, \ldots
$$

Therefore, $\left\{y_{n}\right\}_{n=1}^{\infty}$ exists the sub-sequence converges $\left\{y_{n_{i}}\right\}_{i=0}^{\infty}$ to $y^{*} \in \Omega$. Because $\left\{y_{n_{i}}\right\}_{i=0}^{\infty}$ is monotonic, and $y_{n} \rightarrow y^{*}$, again from the continuity of the operator $T$, it can be known that $T y_{n}=y_{n+1} \rightarrow y^{*}$, that is $T y^{*}=y^{*}$. It remains to be seen is that, the problem (3.1) has two positive solutions $x^{*}$ and $y^{*}$. So $\left\|x^{*}\right\|>0$ and $\left\|y^{*}\right\|>0$. The proof is complete. 


\section{Example}

Consider the following problem:

$$
\left\{\begin{array}{c}
D_{\frac{1}{2}}^{\frac{3}{2}}\left(\phi_{\frac{3}{2}}\left(D_{\frac{1}{2}}^{\frac{3}{2}} x(t)\right)\right)=\frac{x^{2}}{15}+\frac{t x}{12}, \quad 0<t<1 \\
x(0)=0, \quad x(1)=\frac{1}{4} D_{\frac{1}{2}}^{\frac{1}{4}} x\left(\frac{1}{2}\right), \quad D_{\frac{1}{2}}^{\frac{3}{2}} x(0)=0, \quad D_{\frac{1}{2}}^{\frac{3}{2}} x(1)=\frac{1}{2} D_{\frac{1}{2}}^{\frac{3}{2}} x\left(\frac{1}{2}\right) .
\end{array}\right.
$$

Where $q=\frac{1}{2}, p=\frac{3}{2}, h(y, x(t))=\frac{x^{2}}{15}+\frac{t x}{12}, \alpha=\beta=\frac{3}{2}, \gamma=\frac{1}{4}, \quad \xi=\frac{1}{2}$, $\eta=\frac{1}{2}, a=\frac{1}{4}, b=\frac{1}{2}$.Then

$$
\begin{gathered}
\Gamma_{q}\left(\frac{\beta+1}{2}\right)-a \Gamma_{q}(\beta) \xi^{\frac{\beta-1}{2}}=\Gamma_{\frac{1}{2}}\left(\frac{5}{4}\right)-\frac{1}{4} \times \Gamma_{\frac{1}{2}}\left(\frac{3}{2}\right) \times\left(\frac{1}{2}\right)^{\frac{1}{4}}>0, \\
1-b^{p-1} \eta^{\alpha-1}=1-\left(\frac{1}{2}\right)^{\frac{1}{2}} \times\left(\frac{1}{2}\right)^{\frac{1}{2}}=0.5>0, \\
J=\left(\int_{0}^{1} \mu(q z) \phi_{q^{*}}\left(\int_{0}^{1} v(q r) d_{q} r\right) d_{q} z\right)^{-1} \approx 4.3367 .
\end{gathered}
$$

Let $k=8$, we have

(1) For $\forall 0 \leq t \leq 1$, one has $0 \leq s_{1} \leq s_{2} \leq 8, h\left(t, s_{1}\right) \leq h\left(t, s_{2}\right)$;

(2) $\max _{0 \leq t \leq 1} h(t, k)=h(1,8) \approx 4.9334<\phi_{p}(k J) \approx 5.8901$;

(3) $h(t, 0)=0$, for $0 \leq t \leq 1$.

The problem (9) has two positive solutions $x^{*}$ and $y^{*}$ :

(i) $0<\left\|x^{*}\right\| \leq 8$ and $\lim _{n \rightarrow \infty} T^{n} x_{0}=x^{*}$,where $x_{0}(t)=8$;

(ii) $0<\left\|y^{*}\right\| \leq 9$ and $\lim _{n \rightarrow \infty} T^{n} y_{0}=y^{*}$, where $y_{0}(t)=0$.

\section{References}

[1] Annaby M H and Mansour Z S 2012 Q-difference equations Lecture Notes in Mathematics

[2] Fan X, Zhang Q and Zhao D 2005 Eigenvalues of p(x)-Laplacian dirichlet problem Journal of Mathematical Analysis and Applications 302(2) 306-17 
[3] Wang J and Xiang H 2014 Upper and lower solutions method for a class of singular fractional boundary value problems with p-laplacian operator Abstract \& Applied Analysis 2010 1085-3375

[4] Bai and Chuan Z 2018 Existence and uniqueness of solutions for fractional boundary value problems with p-laplacian operator Advances in Difference Equations 2018(1) 4-10

[5] Zhou B 2019 Existence of positive solutions of boundary value problems for high-order nonlinear conformable differential equations with p-Laplacian operator Adv. Differ. Equ. 351 34-44

[6] Lu H, Han Z, Chao Z and Yan Z 2014 Positive Solutions for Boundary Value Problem of Nonlinear Fractional Differential Equation with p-Laplacian Operator Journal of Differential Equations 2014

[7] Rao S N and Alesemi M 2020 Existence of positive solutions for systems of nonlinear fractional differential equation with p-laplacian Asian-European Journal of Mathematics 13(05) 5-719

[8] Guo L and Liu L 2019 Unique iterative positive solutions for a singular p-laplacian fractional differential equation system with infinite-point boundary conditions. Boundary Value Problems 1

[9] Li Y 2019 Multiple positive solutions for nonlinear mixed fractional differential equation with p-laplacian operator Advances in Difference Equations 1

[10] Khan H, Jarad F, Abdeljawad T and Khan A. A 2019 Singular abc-fractional differential equation with p-laplacian operator Chaos, Solitons \& Fractals 129

[11] Jong K S, Choi H C and Ri Y H 2019 Existence of positive solutions of a class of multi-point boundary value problems for p-laplacian fractional differential equations with singular source terms Communications in Nonlinear Science and Numerical Simulation 72(JUN.) 272-81

[12] Zhang L, Wang F and RuY 2019 Existence of nontrivial solutions for fractional differential equations with p-laplacian Journal of Function Spaces 2019 1-12

[13] Hka B, Fj C, Ta D and Ak D 2019 A singular abc-fractional differential equation with p -laplacian operator. Chaos Solitons \& Fractals 129 56-61

[14] Haddouchi F 2019 Positive solutions of p-laplacian fractional differential equations with fractional derivative boundary condition 2019

[15] Chen T and Liu W Z 2012 A boundary value problem for fractional differential equation with p-Laplacian operator at resonance Nonlinear Analysis Theory Methods and Applications 2012

[16] Bartsch T and Liu Z 2014 On a superlinear elliptic p-laplacian equation Journal of Differential Equations 198(1) $149-75$

[17] Lu S and Gui Z 2007 On the existence of periodic solutions to p-laplacian rayleigh differential equation with a delay Journal of Mathematical Analysis \& Applications 325(1) 685-702

[18] Klaus P 1998 Existence and multiplicity of solutions to a p-laplacian equation with nonlinear boundary condition Electronic Journal of Differential Equations 10 1-13

[19] Mao J, Zhao Z and Wang C 2019 The unique iterative positive solution of fractional boundary value problem with q-difference Applied Mathematics Letters 100 106-200

[20] El-Shahed M and Al-Askar F M 2011 Positive Solutions for Boundary Value Problem of Nonlinear Fractional q-Difference Equation Isrn Mathematical Analysis 11 5545-50

[21] Tsa C, Gs A and Bq B 2020 On the fuzzy difference equation xn = F( xn1, xnk ) Fuzzy Sets and Systems $38781-8$

[22] Gefter S L and Piven A L 2020 Holomorphic Solutions to Linear q-Difference Equations in a Banach Space Journal of Mathematical Sciences D 251(5) 602-614

[23] Abbas S, Benchohra M, LaleDj N, et al 2019 Existence and Ulam stability for implicit fractional q-difference equations. Advances in Difference Equations 1 1-12

[24] Tua H and Gu Y 2020 On the meromorphic solutions of generalized q-difference equations Science Asia 46(5) 626

[25] Kang S, Chen H, Li L et al 2019 Existence of three positive solutions for a class of Riemann-Liouville fractional q-difference equation Journal of Applied Analysis \& Computation 9(2) 590-600

[26] Annaby M H and Mansour Z S 2012 Q-fractional calculus and equations Springer Berlin Heidelberg 2012

[27] Rui A 2010 Nontrivial solutions for fractional q-difference boundary value problems Electronic Journal of Qualitative Theory of Differential Equations 70 1-10

[28] Zhao Y, Chen H and Zhang Q 2013 Existence and multiplicity of positive solutions for nonhomogeneous boundary value problems with fractional q-derivatives Boundary Value Problems 1103

[29] Lian H, Wong P and Shu Y 2015 Solvability of Three-Point Boundary Value Problems at Resonance with a p-Laplacian on Finite and Infinite Intervals Abstract \& Applied Analysis 6 1395-416 